1

2

3

\title{
Fast and Accurate Algorithms for Simulating Coarsening Dynamics of Cahn-Hilliard Equations
}

\author{
Lili Ju ${ }^{\mathrm{a}}$, Jian Zhang ${ }^{\mathrm{b}}$, Qiang Duc,* \\ ${ }^{a}$ Department of Mathematics, University of South Carolina, Columbia, SC 29208, USA. \\ ${ }^{b}$ Computer Network Information Center, Chinese Academy of Sciences, Beijing 100080, China. \\ ${ }^{c}$ Department of Applied Physics and Applied Mathematics, Columbia University, NY 10027, and Department of \\ Mathematics, Pennsylvania State University, University Park, PA 16802, USA.
}

\begin{abstract}
Numerical simulation of microstructure coarsening is a subject of great interest in computational materials science. The coarsening dynamics in a binary mixture can be modeled by the celebrated Cahn-Hilliard equations. To perform efficient and accurate long-time integrations, we develop a fast and stable high order numerical method for solving Cahn-Hilliard equations. The spatial discretization is carried out by the compact central difference scheme with FFT-based fast implementation while the time integration is done through the accurate exponential time differencing multistep approach. We demonstrate the effectiveness of the proposed method by numerical experiments and study computationally the coarsening kinetics corresponding to different choices of the diffusion mobility.
\end{abstract}

Keywords: Phase field model, Cahn-Hilliard equation, variable mobility, coarsening rate, fast Fourier transform

\section{Introduction}

Coarsening dynamics of microstructure refers to a kinetic process driven by the reduction in the total interfacial energy. Understand its mechanism and long-time statistics are of much importance to the study of mechanical properties of a material. There have been many studies of coarsening dynamics, such as the so-called LSW theory [1,2]. For a binary system, Cahn-Hilliard equations [3] are well-known phase field models that have often been used to analyze and simulate coarsening dynamics. For examples, asymptotic analysis of coarsening rates has been given by $[4,5,6,7]$ and numerical simulations can be found in $[8,9,10,11,12,13,14]$. Further theoretical justifications have been given by [15]. It is often observed in numerical simulations and substantiated analytically that some characteristic length scale increases as a power of time, and such power law depends on the diffusion mobility.

\footnotetext{
${ }^{\text {th }} \mathrm{L}$. Ju's research is partially supported by the US National Science Foundation under grant number DMS-1215659 and the U.S. Department of Energy under grant number DE-SC0008087-ER65393. J. Zhang's research is partially supported by the Natural Science Foundation of China under grant numbers 11271350 and 91130019. Q. Du's research is partially supported by the US National Science Foundation under grant number DMS-1318586.

${ }^{*}$ Corresponding author

Email addresses: ju@math.sc.edu (Lili Ju), zhangjian@sccas.cn (Jian Zhang), qd2125@columbia.edu (Qiang $\mathrm{Du})$
} 
Numerical simulations based on the Cahn-Hilliard models have remained time-consuming. Fast high order and efficient spatial discretizations have been widely used ranging from spectral discretizations [16], multigrid algorithms [17] and moving mesh implementations [18, 19, 20]. High order methods in both space and time are shown to be computationally more competitive for phase field simulations even in the sharp interface limit [21]. Thus, high order accurate and stable time discretizations of Cahn-Hilliard equations are desirable for large scale and long-time simulations of coarsening dynamics.

In this work, we develop a fast and stabilized compact exponential time differencing multistep method for solving the Cahn-Hilliard equation in a rectangular domain. Our time stepping method is explicit in nature and thus free from the need to solve linear and nonlinear systems. It utilizes accurate exponential time differences with multistep approximations to maintain accuracy and linear splitting schemes to achieve stability. Compact representation of central difference is used for discretizations of spatial operators so that our method can deal with various boundary conditions and take advantage of Fast Fourier Transform (FFT) based computations. All these techniques are seamlessly coupled together to produce fast, accurate and stable numerical algorithms. We demonstrate the effectiveness of the proposed algorithm by numerical experiments and study computationally the coarsening kinetics corresponding to different choices of the diffusion mobility.

\subsection{Cahn-Hilliard equations}

The Cahn-Hilliard equation considered in this paper takes on the form:

$$
\frac{\partial u}{\partial t}=\nabla \cdot\left(M(u) \nabla\left(-\gamma \Delta u+u^{3}-u\right)\right), \quad \mathbf{x} \in \Omega, t \in\left[t_{0}, t_{0}+T\right]
$$

where $\Omega$ is the spatial material domain in $\mathbb{R}^{d}, t_{0}$ is the initial time and $T>0$ is the time duration of interest. The function $M(u) \geq 0$ is the diffusion mobility while the parameter $\epsilon=\sqrt{\gamma}$ gives the typical length of the diffuse interface transition regions between the phase domains, and $\mu=$ $-\gamma \Delta u+u^{3}-u$ is the chemical potential. The free energy associated with the Cahn-Hilliard equation is often identified as

$$
\mathcal{E}(u)=\int_{\Omega} \frac{1}{4}\left(u^{2}-1\right)^{2}+\frac{\gamma}{2}|\nabla u|^{2} d \mathbf{x} .
$$

The equation (1) is provided with an initial condition $u(x, t)=u_{0}(x)$. Boundary conditions, such as periodic or no-flux boundary conditions, are typically imposed on both $u$ and $\mu$ in most existing simulations. Under these boundary conditions, it can be shown that

$$
\frac{d \mathcal{E}}{d t}=-\int_{\Omega}|\nabla \mu|^{2} d \mathbf{x} \leq 0
$$

corresponding to the reduction of energy $\mathcal{E}(u)$ in time.

\section{Fast and stabilized compact exponential time differencing multistep method}

Traditionally, most of the numerical simulations of phase field equations have been carried out by explicit time marching based on the Euler scheme. The use of semi-implicit and time-splitting schemes have led to significant speed up and improvement of accuracy[16, 17, 20, 22, 23, 24]. The use of exponential integrators for time integration in the solution of semilinear parabolic equations, 
including methods like the classic integrating factor (IF) and the exponential time differencing (ETD) $[25,26,27,28]$, have attracted much attention in the past several decades. They both can maintain the exponential behaviors and differ mainly in the way of evaluating the time integration of the terms formed by multiplication of the exponential integrator and the nonlinear part of the target equation. The classic integrating factor methods often apply quadrature rules directly to the whole integrand, while the ETD-based methods instead first approximate the nonlinear part using polynomial interpolations and then perform exact integrations on the resulting new integrands. Therefore, the ETD-based methods are usually much more accurate due to highly different decaying rates of the exponential integrator components, and thus are very popular and suitable for developing explicit time-stepping schemes. Some simple ETD schemes have been used for phase field simulations [29]. A nice review and additional references on the exponential integrator based methods can be found in [30].

In the following, we present a fast and stabilized compact ETD multistep method for solving the model problem (1) in two dimensions and the proposed algorithms can be straightforwardly extended to three-dimensional space.

\subsection{The constant mobility problem with periodic boundary condition}

We first consider the Cahn-Hilliard equation with a constant mobility, i.e., $M(u) \equiv D>0$ in the equation (1):

$$
\frac{\partial u}{\partial t}=D\left(-\gamma \Delta^{2} u+\Delta\left(u^{3}-u\right)\right)
$$

Let us first apply a linear splitting scheme commonly used in the stabilized semi-implicit methods for energy stabilization $[16,20]$ and rewrite (4) as follows:

$$
\frac{\partial u}{\partial t}=-D\left(\gamma \Delta^{2} u-\kappa \Delta u\right)+\hat{f}(u)
$$

where $\hat{f}(u)=D \Delta\left(u^{3}-(\kappa+1) u\right)$ and $\kappa$ is a splitting constant parameter. It is required that at least

$$
\kappa \geq \frac{1}{2} \max _{u \in[-1,1]}\left\{0,3 u^{2}-1\right\}=1 .
$$

Larger $\kappa$ gives better stability but may lead to larger splitting errors [29, 33]. We will take $\kappa=2$ in the numerical experiments to enforce the energy stability.

Denote the rectangular domain by $\Omega=\left\{x_{b}<x<x_{e}, y_{b}<y<y_{e}\right\}$. We first assume that periodic boundary conditions

$$
\left\{\begin{array}{lc}
u\left(t, x_{b}, y\right)=u\left(t, x_{e}, y\right), u_{x}\left(t, x_{b}, y\right)=u_{x}\left(t, x_{e}, y\right), & y \in\left[y_{b}, y_{e}\right], t \in\left[t_{0}, t_{0}+T\right], \\
u\left(t, x, y_{b}\right)=u\left(t, x, y_{e}\right), u_{y}\left(t, x, y_{b}\right)=u_{y}\left(t, x, y_{e}\right), & x \in\left[x_{b}, x_{e}\right], t \in\left[t_{0}, t_{0}+T\right], \\
\mu\left(t, x_{b}, y\right)=\mu\left(t, x_{e}, y\right), \mu_{x}\left(t, x_{b}, y\right)=\mu_{x}\left(t, x_{e}, y\right), & y \in\left[y_{b}, y_{e}\right], t \in\left[t_{0}, t_{0}+T\right], \\
\mu\left(t, x, y_{b}\right)=\mu\left(t, x, y_{e}\right), \mu_{y}\left(t, x, y_{b}\right)=\mu_{y}\left(t, x, y_{e}\right), & x \in\left[x_{b}, x_{e}\right], t \in\left[t_{0}, t_{0}+T\right]
\end{array}\right.
$$

are imposed, which also imply the periodicity in $\Delta u$ by simple derivation.

Let us discretize the spatial domain $\Omega$ by a rectangular mesh as follows: $\left(x_{i}, y_{j}\right)=\left(x_{b}+\right.$ $\left.i h_{x}, y_{b}+j h_{y}\right)$ where $h_{x}=\left(x_{e}-x_{b}\right) / N_{x}, h_{y}=\left(y_{e}-y_{b}\right) / N_{y}$ and $0 \leq i \leq N_{x}$ and $0 \leq j \leq N_{y}$. Set $u_{i, j} \approx u\left(t, x_{i}, y_{j}\right)$ and $\Delta u_{i, j} \approx \Delta u\left(t, x_{i}, y_{j}\right)$ for $0 \leq i \leq N_{x}$ and $0 \leq j \leq N_{y}$. Denote the set of unknowns as $\mathbf{U}=\left(u_{i-1, j-1}\right)_{1 \leq i \leq N_{x}, 1 \leq j \leq N_{y}}$. 
Let $\mathbf{G}_{M}^{P}$ denote a symmetric tri-diagonal $M \times M$ matrix such that $\left(\mathbf{G}_{M}^{P}\right)_{i, i}=-2$ for $i=$ $1, \cdots, M,\left(\mathbf{G}_{M}^{P}\right)_{i, i-1}=1$ for $i=2, \cdots, M,\left(\mathbf{G}_{M}^{P}\right)_{i, i+1}=1$ for $i=1, \cdots, M-1,\left(\mathbf{G}_{M}^{P}\right)_{1, M}=$ $\left(\mathbf{G}_{M}^{P}\right)_{M, 1}=1$, and $\left(\mathbf{G}_{M}^{P}\right)_{i, j}=0$ otherwise. Set

$$
\mathbf{A}=\frac{1}{h_{x}^{2}} \mathbf{G}_{N_{x}}^{P}, \quad \mathbf{B}=\frac{1}{h_{y}^{2}} \mathbf{G}_{N_{y}}^{P} .
$$

We further define two special operators as follows: for any $M \times M$ matrix $U, \mathbf{A} \times \mathbf{U}=\mathbf{A} \mathbf{U}$ and $\mathbf{B}$ (4) $\mathbf{U}=\mathbf{U B}$, that is

$$
(\mathbf{A} \circledast \mathbf{U})_{i, j}=\sum_{l=1}^{N_{x}}(\mathbf{A})_{i, l}(\mathbf{U})_{l, j}, \quad\left(\mathbf{B}(\mathbf{y} \mathbf{U})_{i, j}=\sum_{l=1}^{N_{y}}(\mathbf{B})_{j, l}(\mathbf{U})_{i, l} .\right.
$$

Note that these two operators are commutative, i.e., $\mathbf{B}(\mathbf{A} \circledast \mathbf{U}=\mathbf{A} \circledast \mathbf{B} \bigoplus \mathbf{U}$, where by convention, $\mathbf{B}(y) \mathbf{A}(\mathbf{U}$ means $\mathbf{B}(y)(\mathbf{A}(\mathbf{U})$ and $\mathbf{A} \circledast \mathbf{B}(y) \mathbf{U}=\mathbf{A}(x)(\mathbf{B}(y) \mathbf{U})$. By using the second-order accurate central difference discretization scheme for the spatial derivatives, we then obtain a semidiscretization of (4) in space in the following compact representation:

$$
\frac{d \mathbf{U}}{d t}=-D\left[\gamma\left(\mathbf{A}^{2} \circledast \mathbf{U}+2 \mathbf{B}\left(\mathbf{A} \circledast \mathbf{U}+\mathbf{B}^{2}(y) \mathbf{U}\right)-\kappa(\mathbf{A} \circledast \mathbf{U}+\mathbf{B}(y) \mathbf{U})\right]+\mathcal{F}(\mathbf{U}),\right.
$$

where $\mathbf{A}^{2}=\mathbf{A} \mathbf{A}$ and $\mathbf{B}^{2}=\mathbf{B B}$ are regular matrix multiplications and

$$
\mathcal{F}(\mathbf{U})=D\left[\mathbf{A} \circledast\left(\mathbf{U}^{* 3}-(1+\kappa) \mathbf{U}\right)+\mathbf{B}(4)\left(\mathbf{U}^{* 3}-(1+\kappa) \mathbf{U}\right)\right]
$$

with $\mathbf{U}^{* 3}=\left(u_{i-1, j-1}^{3}\right)_{N_{x} \times N_{y}}$.

It is easy to find that in this case $\mathbf{A}$ and $\mathbf{B}$ are diagonalizable and it holds that

$$
\mathbf{A}=\mathbf{P}_{x} \mathbf{D}_{x} \mathbf{P}_{x}^{-1}, \quad \mathbf{B}=\mathbf{P}_{y} \mathbf{D}_{y} \mathbf{P}_{y}^{-1}
$$

where $\mathbf{D}_{x}=\operatorname{diag}\left[d_{1}^{x}, \cdots, d_{N_{x}}^{x}\right], \mathbf{D}_{y}=\operatorname{diag}\left[d_{1}^{y}, \cdots, d_{N_{y}}^{y}\right]$ with

$$
\begin{array}{ll}
d_{k}^{x}=-\frac{4}{h_{x}^{2}} \sin ^{2}\left(\frac{(k-1) \pi}{N_{x}}\right), \quad\left(\mathbf{P}_{x}\right)_{k, j}=\exp \left(-\mathrm{i} \frac{2 \pi(k-1)(j-1)}{N_{x}}\right), \quad k, j=1, \cdots, N_{x}, \\
d_{k}^{y}=-\frac{4}{h_{y}^{2}} \sin ^{2}\left(\frac{(k-1) \pi}{N_{y}}\right), \quad\left(\mathbf{P}_{y}\right)_{k, j}=\exp \left(-\mathrm{i} \frac{2 \pi(k-1)(j-1)}{N_{y}}\right), \quad k, j=1, \cdots, N_{y},
\end{array}
$$

where $\mathrm{i}=\sqrt{-1}$. By using the substitution $\mathbf{V}=\mathbf{P}_{y}^{-1}(y) \mathbf{P}_{x}^{-1} \cong \mathbf{U}$, we can tranform (9) can be into

$$
\frac{d \mathbf{V}}{d t}+D\left[\gamma \left(\mathbf{D}_{x}^{2} \circledast \mathbf{V}+2 \mathbf{D}_{y}\left(\mathbf{D}_{x} \circledast \mathbf{V}+\mathbf{D}_{y}(\mathbf{V})-\kappa\left(\mathbf{D}_{x} \circledast \mathbf{V}+\mathbf{D}_{y}(\mathbf{V})\right]=\mathbf{P}_{y}^{-1}\left(\mathbf{P}_{x}^{-1} \circledast \mathcal{F}(\mathbf{U})\right.\right.\right.\right.
$$

Set $\mathbf{H}=\left(h_{i, j}\right)$ with

$$
h_{i, j}=D\left[\gamma\left(d_{i}^{x}+d_{j}^{y}\right)^{2}-\kappa\left(d_{i}^{x}+d_{j}^{y}\right)\right] \geq 0,
$$

for $i=1,2, \cdots N_{x}, j=1,2, \cdots N_{y}$. Define the operation " $e$ " to be taking exponentials of a matrix element by element as

$$
\left(e^{*}\right)^{\mathbf{H}}=\left(e^{h_{i, j}}\right)_{N_{x} \times N_{y}},
$$


and the operator " $\odot$ " for element by element multiplication between two matrices of same sizes as

$$
(\mathbf{M} \odot \mathbf{L})_{i, j}=\left(m_{i, j} l_{i, j}\right),
$$

where $\mathbf{M}=\left(m_{i, j}\right), \mathbf{L}=\left(l_{i, j}\right)$. Then, direct calculations on (11) leads to

$$
\frac{d\left(\mathbf{V} \odot\left(e^{*}\right)^{\mathbf{H} t}\right)}{d t}=\left(\mathbf{P}_{y}^{-1}(y) \mathbf{P}_{x}^{-1} \circledast \mathcal{F}(\mathbf{U})\right) \odot\left(e^{*}\right)^{\mathbf{H} t} .
$$

Let us discretize the time interval of interest as $t_{n}=t_{0}+n \Delta t, n=0,1, \cdots, N_{t}$ with a uniform time step size $\Delta t=T / N_{t}$. Then integrating on both sides of (12) from $t_{n}$ to $t_{n}+1$ gives us

$$
\mathbf{V}_{n+1}=\left(\mathbf{V}_{n}+\int_{0}^{\Delta t}\left(\mathbf{P}_{y}^{-1}(4) \mathbf{P}_{x}^{-1} \circledast \mathcal{F}\left(\mathbf{U}\left(t_{n}+\tau\right)\right)\right) \odot\left(e^{*}\right)^{\mathbf{H} \tau} d \tau\right) \odot\left(e^{*}\right)^{-\mathbf{H} \Delta t} .
$$

Finally we obtain a general approximation for solving $\mathbf{U}$ from (14) as

$$
\begin{aligned}
\mathbf{U}_{n+1}=\mathbf{P}_{y}(y) \mathbf{P}_{x} \circledast( & \left(\mathbf{P}_{y}^{-1}(y) \mathbf{P}_{x}^{-1} \circledast \mathbf{U}_{n}\right) \odot\left(e^{*}\right)^{-\mathbf{H} \Delta t}+\int_{0}^{\Delta t}\left(\mathbf{P}_{y}^{-1}\left(y \mathbf{P}_{x}^{-1} \circledast \mathcal{F}\left(\mathbf{U}\left(t_{n}+\tau\right)\right)\right)\right. \\
& \left.\odot\left(e^{*}\right)^{-\mathbf{H}(\Delta t-\tau)} d \tau\right)
\end{aligned}
$$

Next we take an explicit multi-step approach to evaluate the integral on the right-hand side. We use the Lagrange polynomial interpolation of degree $r$ to approximate

$$
\mathbf{F}\left(t_{n}+\tau\right)=\mathbf{P}_{y}^{-1}(y) \mathbf{P}_{x}^{-1} \circledast \mathcal{F}\left(\mathbf{U}\left(t_{n}+\tau\right)\right) .
$$

Define

$$
P_{r}(\tau)=\sum_{s=0}^{r} \mathbf{F}\left(t_{n-s}\right) \eta_{r, s}(\tau), \quad 0 \leq \tau \leq \Delta t,
$$

where $\eta_{r, s}(\tau)=\prod_{l=0, l \neq s}^{r} \frac{t_{n}+\tau-t_{n-l}}{t_{n-s}-t_{n-l}}$. We have the interpolation error $\mathbf{F}(t+\tau)-P_{r}(\tau)=O\left((\Delta t)^{r+1}\right)$ if the derivative $\left|\frac{d^{r+1} \mathbf{F}(t)}{d t^{r+1}}\right|$ is bounded. Then we can approximate

$$
\int_{0}^{\Delta t}\left(\mathbf{F}\left(t_{n}+\tau\right)\right)_{i, j} e^{-h_{i, j}(\Delta t-\tau)} d \tau \approx \sum_{s=0}^{r}\left(\mathbf{F}\left(t_{n-s}\right)\right)_{i, j} \beta_{i, j}^{r, s}
$$

with $\beta_{i, j}^{r, s}=\int_{0}^{\Delta t} e^{-h_{i, j}(\Delta t-\tau)} \eta_{r, s}(\tau) d \tau$. Remark that $\left\{\beta_{i, j}^{r, s}\right\}_{s=0}^{r}$ does not depend on time for uniform time partition. For simplicity, we only present the values of $\alpha_{i, j}^{r_{1}, s}$ and $\beta_{i, j}^{r_{2}, s}$ for $r=0,1,2$ below:

$$
\begin{cases}\beta_{i, j}^{0,0}=\phi_{0} ; & \beta_{i, j}^{1,1}=-\phi_{1} ; \\ \beta_{i, j}^{1,0}=\phi_{0}+\phi_{1}, & \beta_{i, j}^{2,1}=-\left(2 \phi_{1}+\phi_{2}\right), \quad \beta_{i, j}^{2,2}=\frac{\phi_{1}+\phi_{2}}{2} ; \\ \beta_{i, j}^{2,0}=\frac{1}{2}\left(2 \phi_{0}+3 \phi_{1}+\phi_{2}\right)\end{cases}
$$

where

$$
\begin{cases}\phi_{0}=\frac{1}{h_{i, j}}\left(1-e^{-h_{i, j} \Delta t}\right), \phi_{1}=\frac{1}{h_{i j}}\left(1-\frac{\phi_{0}}{\Delta t}\right), \phi_{2}=\frac{1}{h_{i j}}\left(1-\frac{2 \phi_{1}}{\Delta t}\right), & h_{i, j} \neq 0, \\ \phi_{0}=\Delta t, \quad \phi_{1}=\frac{\Delta t}{2}, \quad \phi_{2}=\frac{\Delta t}{3}, & h_{i, j}=0 .\end{cases}
$$


Define $\mathbf{S}_{r, s}=\left(\beta_{i, j}^{r, s}\right)_{N_{x} \times N_{y}}$, then we have the following approximations:

$$
\int_{0}^{\Delta t} \mathbf{F}\left(t_{n}+\tau\right) \odot\left(e^{*}\right)^{-\mathbf{H}(\Delta t-\tau)} d \tau \approx \sum_{s=0}^{r} \mathcal{F}\left(\mathbf{U}_{n-s}\right) \odot \mathbf{S}_{r, s} .
$$

Based on (14) and (17), we then obtain the following fully discrete stabilized compact ETD multistep (cETDMs) method for solving the constant mobility Cahn-Hilliard equation (4) as

$$
=\mathbf{P}_{y}(y) \mathbf{P}_{x} \circledast\left[\left(\mathbf{P}_{y}^{-1}\left(y \mathbf{P}_{x}^{-1} \circledast \mathbf{U}_{n}\right) \odot\left(e^{*}\right)^{-\mathbf{H} \Delta t}+\sum_{s=0}^{r}\left(\mathbf{P}_{y}^{-1}\left(y \mathbf{P}_{x}^{-1} \circledast \mathcal{F}\left(\mathbf{U}_{n-s}\right)\right) \odot \mathbf{S}_{r, s}\right] .\right.\right.
$$

This scheme is expected to be second-order accurate in space and $(r+1)$-th order in time.

Remark 1. Let $\mathbf{V}$ be any $N_{x} \times N_{y}$ array, we can verify that $\mathbf{P}_{x}(\mathbf{V}$ means applying a Discrete Fourier Transform (DFT) to each column of $\mathbf{V}$ and $\mathbf{P}_{x}^{-1} \circledast \mathbf{V}$ does an inverse DFT, i.e.,

$$
\mathbf{P}_{x} \circledast \mathbf{V}=\operatorname{DFT}_{N_{x}}(\mathbf{V}), \quad \mathbf{P}_{x}^{-1} \circledast \mathbf{V}=\mathrm{iDFT}_{N_{x}}(\mathbf{V}) .
$$

Similarly, $\mathbf{P}_{y}$ (4) V means applying a DFT to each row of $\mathbf{V}$ and $\mathbf{P}_{y}^{-1}(4) \mathbf{V}$ does an inverse DFT, i.e.,

$$
\mathbf{P}_{y}(y) \mathbf{V}=\operatorname{DFT}_{N_{y}}\left(\mathbf{V}^{T}\right)^{T}, \quad \mathbf{P}_{y}^{-1} \text { (y) } \mathbf{V}=\operatorname{iDFT}_{N_{y}}\left(\mathbf{V}^{T}\right)^{T} \text {. }
$$

Thus the computational complexity of the scheme (18) is $O\left(N^{2} \log _{2} N\right)$ per time step where $N=$ $\max \left\{N_{x}, N_{y}\right\}$ through the use of FFT [33].

\subsection{The variable mobility problem with periodic boundary condition}

Now we consider the model problem (1) with variable mobility, for example, $M(u)=1-a u^{2}$ (or $\left.M(u)=\left|1-a u^{2}\right|\right)$ and $M(u)=(1+a u) / 2($ or $M(u)=|1+a u| / 2)$ with $0 \leq a \leq 1[6,7,31,32,12]$. We first transform the Cahn-Hilliard equation (1) into the following form:

$$
\frac{\partial u}{\partial t}=-C\left(\gamma \Delta^{2} u\right)-\nabla \cdot((M(u)-C) \nabla(\gamma \Delta u))+\nabla \cdot\left(M(u) \nabla\left(u^{3}-u\right)\right),
$$

where $C>0$ is a splitting parameter used to control $|M(u)-C|$. In numerical experiments we take at each time step

$$
C=\max _{i, j} M\left((\mathbf{U})_{i, j}\right) .
$$

It is easy to check that $C \geq C-M\left((\mathbf{U})_{i, j}\right) \geq 0$ under such setting since $M\left((\mathbf{U})_{i, j}\right) \geq 0$. We specially note that our selection for the value of $C$ here is different from that suggested in [16] where $C=\frac{1}{2}\left[\max _{i, j} M\left((\mathbf{U})_{i, j}\right)+\min _{i, j} M\left((\mathbf{U})_{i, j}\right)\right]$.

To apply the linear splitting technique for energy stabilization as in the constant mobility case, we further rewrite (19) as

$$
\frac{\partial u}{\partial t}=-C\left(\gamma \Delta^{2} u-\kappa \Delta u\right)+\tilde{f}(u)
$$

where $\tilde{f}(u)=\tilde{f}_{1}(u)+\tilde{f}_{2}(u)$ with

$$
\tilde{f}_{1}(u)=C \Delta\left(u^{3}-(1+\kappa) u\right), \quad \tilde{f}_{2}(u)=\nabla \cdot\left((M(u)-C) \nabla\left(-\gamma \Delta u+u^{3}-u\right)\right) .
$$


Let us approximate $\tilde{f}_{1}(u)$ pointwisely using second-order central finite difference by

$$
\mathcal{F}_{1}(\mathbf{U})=C\left[\mathbf{A} \circledast\left(\mathbf{U}^{* 3}-(1+\kappa) \mathbf{U}\right)+\mathbf{B}(4)\left(\mathbf{U}^{* 3}-(1+\kappa) \mathbf{U}\right)\right] .
$$

The approximation of $\tilde{f}_{2}(u)$ is more complicated since $M(u)$ is variable. We instead use a secondorder accurate central finite volume discretization for its discretization. Set

$$
\mathbf{E}=-\gamma\left(\mathbf{A} \circledast \mathbf{U}+\mathbf{B}(\mathbf{U})+\mathbf{U}^{* 3}-\mathbf{U}, \quad(\mathbf{L})_{i, j}=M\left(u_{i, j}\right)-C,\right.
$$

then we can approximate $\tilde{f}_{2}(u)$ by

$$
\begin{gathered}
\left(\mathcal{F}_{2}(\mathbf{U})\right)_{i, j}=c_{l}(\mathbf{E})_{i_{l}, j}+c_{r}(\mathbf{E})_{i_{r}}+c_{b}(\mathbf{E})_{i, j_{l}}+c_{t}(\mathbf{E})_{i, j_{r}}-\left(c_{l}+c_{r}+c_{b}+c_{t}\right)(\mathbf{E})_{i, j}, \\
i=1,2, \cdots, N_{x}, j=1,2 \cdots, N_{y},
\end{gathered}
$$

where

$$
\begin{gathered}
i_{l}=\bmod \left(i-2, N_{x}\right)+1, i_{r}=\bmod \left(i, N_{x}\right)+1, j_{l}=\bmod \left(j-2, N_{y}\right)+1, j_{r}=\bmod \left(j, N_{y}\right)+1, \\
c_{l}=\frac{(\mathbf{L})_{i_{l}, j}+(\mathbf{L})_{i, j}}{2 h_{x}^{2}}, \quad c_{r}=\frac{(\mathbf{L})_{i_{r}, j}+(\mathbf{L})_{i, j}}{2 h_{x}^{2}}, \quad c_{b}=\frac{(\mathbf{L})_{i, j_{l}}+(\mathbf{L})_{i, j}}{2 h_{y}^{2}}, \quad c_{t}=\frac{(\mathbf{L})_{i, j_{r}}+(\mathbf{L})_{i, j}}{2 h_{y}^{2}} .
\end{gathered}
$$

We then obtain a semi-discretization in space of (21) in the following compact representation:

$$
\frac{d \mathbf{U}}{d t}=-C\left[\gamma\left(\mathbf{A}^{2} \circledast \mathbf{U}+2 \mathbf{B}(y) \mathbf{A} \circledast \mathbf{U}+\mathbf{B}^{2}(y) \mathbf{U}\right)-\kappa(\mathbf{A} \circledast \mathbf{U}+\mathbf{B}(y \mathbf{U})]+\mathcal{F}(\mathbf{U}),\right.
$$

with $\mathcal{F}(\mathbf{U})=\mathcal{F}_{1}(\mathbf{U})+\mathcal{F}_{2}(\mathbf{U})$, that is in the same form of (9). Then a compact ETD multistep solution method for solving the variable mobility Cahn-Hilliad equation (1) is identical to (18) except now $\mathbf{H}=\left(h_{i, j}\right)$ with $h_{i, j}=C\left[\gamma\left(d_{i}^{x}+d_{j}^{y}\right)^{2}-\kappa\left(d_{i}^{x}+d_{j}^{y}\right)\right]$.

\subsection{No-flux boundary condition}

Now we consider the Cahn-Hilliard equation problem (1) imposed with no-flux boundary condition as

$$
\frac{\partial u}{\partial \mathbf{n}}=\frac{\partial \mu}{\partial \mathbf{n}}=0, \quad \mathbf{x} \in \partial \Omega, t \in\left[t_{0}, t_{0}+T\right]
$$

which are equivalent to

$$
\frac{\partial u}{\partial \mathbf{n}}=\frac{\partial \Delta u}{\partial \mathbf{n}}=0, \quad \mathbf{x} \in \partial \Omega, t \in\left[t_{0}, t_{0}+T\right] .
$$

In this case we denote $\mathbf{U}=\left(u_{i-1, j-1}\right)_{1 \leq i \leq N_{x}+1,1 \leq j \leq N_{y}+1}$ which has one extra row and column than the periodic case. We obtain $\mathbf{G}_{M}^{N}$ from $\mathbf{G}_{M}^{P}$ by changing the (1,2)-th and $(M, M-1)$-th entries from 1 to 2 , and the $(1, M)$-th and $(M, 1)$-th entries from 1 to to 0 . Similarly set as before

$$
\mathbf{A}=\frac{1}{h_{x}^{2}} \mathbf{G}_{N_{x}+1}^{N}, \quad \mathbf{B}=\frac{1}{h_{y}^{2}} \mathbf{G}_{N_{y}+1}^{N} .
$$

Note the above matrices $\mathbf{A}$ and $\mathbf{B}$ are no longer symmetric. The entries of diagonal matrices $\tilde{\mathbf{D}}_{x}$ and $\tilde{\mathbf{D}}_{y}$ (not eigenvalues of $\mathbf{A}$ and $\mathbf{B}$ anymore), and the operations $\mathbf{P}_{x} \circledast \mathbf{V}, \mathbf{P}_{x}^{-1} \propto \mathbf{V}, \mathbf{P}_{y}(y) \mathbf{V}$, $\mathbf{P}_{y}^{-1}$ (y) $\mathbf{V}$ can be calculated through the use of fast reflective Discrete Fourier Transform (RDFT), 
see [33] for details. Then we again can obtain a stabilized compact ETD multistep method for solving the problem (1) with no-flux boundary condition almost as same as (18). We also note that for the variable mobility problem under the no-flux boundary, the following slight modifications on $i_{l}, i_{r}, j_{l}, j_{r}$ for the calculations of $\mathbf{F}_{2}$ in $(22)$ are needed:

$i_{l}=\left\{\begin{array}{ll}i-1, & i>1 \\ 2, & i=1\end{array}, i_{r}=\left\{\begin{array}{ll}i+1, & i<N_{x}+1 \\ N_{x}, & i=N_{x}+1\end{array}, j_{l}=\left\{\begin{array}{ll}j-1, & j>1 \\ 2, & j=1\end{array}, j_{r}=\left\{\begin{array}{ll}j+1, & j<N_{y}+1 \\ N_{y}, & j=N_{y}+1\end{array}\right.\right.\right.\right.$.

The overall computational complexity of the scheme for the problem with no-flux boundary condition is still $O\left(N^{2} \log _{2} N\right)$ per time step by using FFT.

\section{Numerical experiments}

In this section, we focus on testing the proposed stabilized compact ETD multistep method for solving Cahn-Hilliard equations and simulations of the corresponding coarsening dynamics. We first carry out some experiments to demonstrate the accuracy and temporal convergence of the proposed method measured by the energy and the $L^{2}$ errors. Then we illustrate the effectiveness of the method in simulating the coarsening dynamics by providing accurate estimates of the coarsening rates in long-time phase separation processes corresponding to the different diffusion mobilities.

We consider three models, i.e., the Cahn-Hilliard equation (1) with three different mobilities: a constant mobility $M(u) \equiv 1$, a symmetric degenerate mobility $M(u)=\left|1-u^{2}\right|$ considered in [5], and a one-sided degenerate mobility $M(u)=|1+u| / 2$ (see $[6,7]$ for recent analysis and the references cited therein). The computational domain is taken to be $\Omega=[0,4 \pi]^{2}$ and the model parameter which determines the thickness of the diffusive interface is taken to be $\epsilon=\sqrt{\gamma}=0.02$. A uniform spatial grid with a grid size $h_{x}=h_{y}=h=4 \pi / 2048 \approx 6.135 \times 10^{-3}$ is used to discretize the domain to provide a fine enough spatial resolution.

\subsection{Convergence in time}

We first present convergence tests on the second-order cETDMs scheme (denoted as cETDMs2, i.e. $r=1$ ) scheme and the first-order cETDMs scheme (denoted as cETDMs1, i.e. $r=0$ ), and compare them with a popular stabilized semi-implicit Euler scheme (ss-Euler) for each of the three mobilities. Since the analytic solutions of Cahn-Hilliard equations in general are hard to obtain, for each of the cases, we use the cETDMs2 scheme to run a simulation with a very small time step size and regard the result as the benchmark (the approximate exact solution) which is then used to compute the rates of convergence of the discretization with a larger step size.

Let $\phi$ denote the volume fraction of the two phases. Starting from some fixed configurations (with $\phi=0.5$ for the constant and symmetric degenerate mobilities and $\phi=0.25$ for the one-sided degenerate mobility) as illustrated in Figure 1, we conducted simulations with the time step sizes $\Delta t=6.4 \times 10^{-3}$ to $1 \times 10^{-4}$ (uniformly refined, i.e., $\Delta t=64 \delta, \cdots, 2 \delta, \delta$ with $\delta=1 \times 10^{-4}$ ). For comparative studies, the length of the time interval $T$ was set to be $6.4 \times 10^{-1}$. Corresponding benchmark (approximately exact) solutions were obtained by using a much smaller time step size $\Delta t=2 \times 10^{-5}$, see Figure 2 for some of their surface plots zoomed in $[0, \pi]^{2}$. We tested all schemes on both periodic and no-flux boundary condition cases for all three models, simulation results at the final time were evaluated in terms of errors and convergence rates. All algorithms ran very stably with the tested time step sizes and both types of boundary conditions. 

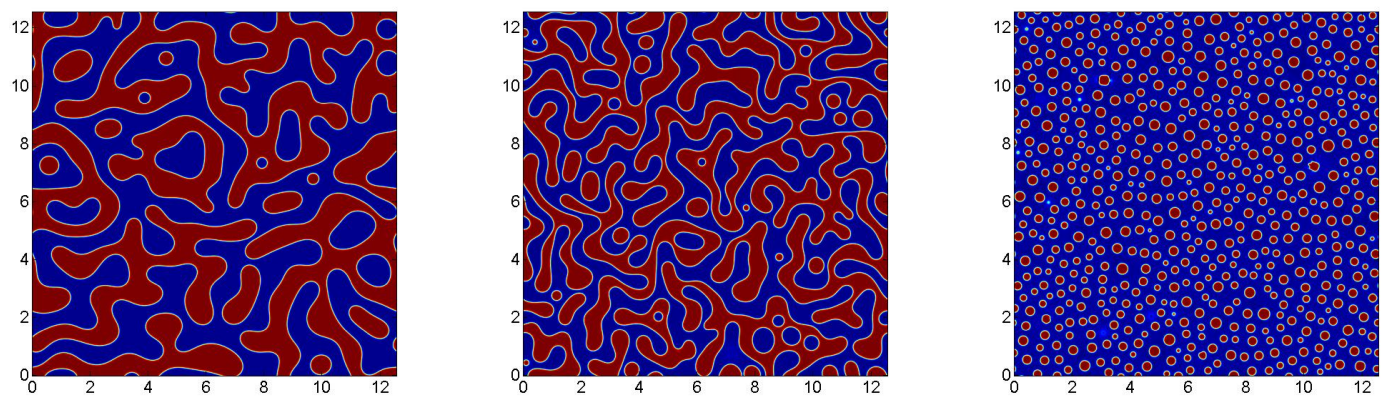

Figure 1: Starting configurations for the temporal convergence tests with periodic BC. From left to right: the Cahn-Hilliard equation with $M(u) \equiv 1, M(u)=\left|1-u^{2}\right|$, and $M(u)=|1+u| / 2$ respectively.
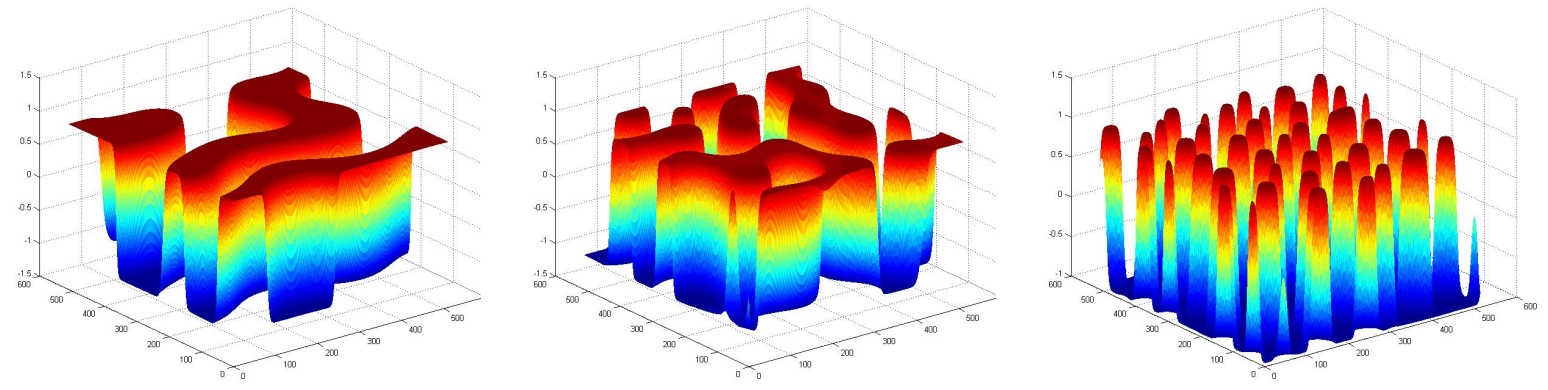

Figure 2: Surface plots of some final configurations of the benchmark soultions for the temporal convergence tests with periodic BC, zoomed in $[0, \pi]^{2}$ (corresponding to the grid $[0,512]^{2}$ ). From left to right: the Cahn-Hilliard equation with $M(u) \equiv 1, M(u)=\left|1-u^{2}\right|$, and $M(u)=|1+u| / 2$ respectively.

\subsubsection{Energy and $L^{2}$ errors}

Numerical results on energy and $L^{2}$ errors obtained with periodic boundary condition are presented in Table 1 (for $M(u) \equiv 1$ ), Table 2 (for $M(u)=\left|1-u^{2}\right|$ ) and Table 3 (for $M(u)=|1+u| / 2$ ). The results with the no-flux boundary condition are very similar and thus not given here. In the tables, the energy error refers to the absolute difference in the energy at the final time while the $L^{2}$ error of the solution refers to the $L^{2}$ norm of the difference in the solution values over space at the final time. The convergence rate is calculated based on the ratio of the error for the current time step size and the error for the preceding larger time step size. Thus no convergence rates are reported for the largest step size.

For the constant mobility problem, it is easy to see that the cETDMs schemes gradually reached their respective optimal temporal convergence rates along the decreasing of time step sizes while the convergence rate of the ss-Euler scheme remained slightly lower than its optimal one for the smallest time step size used here. For the symmetric degenerate mobility problem, the cETDMs schemes showed convergence rates mostly somewhat below their corresponding optimal ones, in particular, around 0.52 to 1.29 for cETDMs1 and 1.45 to 1.72 for cETDMS2 respectively. The ss-Euler scheme was still working as expected in terms of convergence rate but with much larger errors. For the one-sided degenerate mobility problem, all schemes performed quite well in terms of convergence rate, and the cETDMs2 scheme showed some convergence rates of even better than 2 
but with some oscillatory behavior when the errors are measured by the absolute energy difference.

Comparing the errors, we see that the cETDMs1 scheme is more accurate than the ss-Euler scheme and the errors produced by the ss-Euler scheme are usually 1.2 to 5 times of these by the cETDMs1 scheme. More remarkably, the cETDMs2 scheme is superior to both first-order schemes. To achieve almost identical accuracies, we could use tens to hundreds times larger time step sizes in the cETDMs2 scheme than in the cETDMs1 or ss-Euler scheme.

\begin{tabular}{|c||c|c|c|c|c|c|c|}
\hline$\Delta t$ & $6.4 \times 10^{-3}$ & $3.2 \times 10^{-3}$ & $1.6 \times 10^{-3}$ & $8 \times 10^{-4}$ & $4 \times 10^{-4}$ & $2 \times 10^{-4}$ & $1 \times 10^{-4}$ \\
\hline \hline \multicolumn{7}{|c|}{ ss-Euler } \\
\hline Rela. ener. error & $2.828 \mathrm{E}-2$ & $1.945 \mathrm{E}-2$ & $1.217 \mathrm{E}-2$ & $7.094 \mathrm{E}-3$ & $3.949 \mathrm{E}-3$ & $2.138 \mathrm{E}-3$ & $1.135 \mathrm{E}-3$ \\
\hline Conv. rate & - & 0.54 & 0.68 & 0.78 & 0.85 & 0.89 & 0.91 \\
\hline Rela. $L^{2}$ error & $1.533 \mathrm{E}-1$ & $1.128 \mathrm{E}-1$ & $7.434 \mathrm{E}-2$ & $4.456 \mathrm{E}-2$ & $2.510 \mathrm{E}-2$ & $1.366 \mathrm{E}-2$ & $7.288 \mathrm{E}-3$ \\
\hline Conv. rate & - & 0.44 & 0.602 & 0.74 & 0.83 & 0.88 & 0.91 \\
\hline \multicolumn{7}{|c|}{ cETDMs1 } \\
\hline Rela. ener. error & $2.548 \mathrm{E}-2$ & $1.631 \mathrm{E}-2$ & $9.325 \mathrm{E}-3$ & $4.949 \mathrm{E}-3$ & $2.528 \mathrm{E}-3$ & $1.271 \mathrm{E}-3$ & $6.369 \mathrm{E}-4$ \\
\hline Conv. rate & - & 0.64 & 0.81 & 0.91 & 0.97 & 0.99 & 1.00 \\
\hline Rela. $L^{2}$ error & $1.414 \mathrm{E}-1$ & $9.693 \mathrm{E}-2$ & $5.800 \mathrm{E}-2$ & $3.139 \mathrm{E}-2$ & $1.615 \mathrm{E}-2$ & $8.165 \mathrm{E}-3$ & $4.109 \mathrm{E}-3$ \\
\hline Conv. rate & - & 0.55 & 0.74 & 0.89 & 0.96 & 0.98 & 0.99 \\
\hline \multicolumn{7}{|c|}{ cETDMs2 } \\
\hline Rela. ener. error & $8.453 \mathrm{E}-4$ & $2.370 \mathrm{E}-4$ & $6.366 \mathrm{E}-5$ & $1.647 \mathrm{E}-5$ & $4.233 \mathrm{E}-6$ & $1.089 \mathrm{E}-6$ & $2.738 \mathrm{E}-7$ \\
\hline Conv. rate & - & 1.84 & 1.90 & 1.95 & 1.96 & 1.96 & 1.99 \\
\hline Rela. $L^{2}$ error & $6.011 \mathrm{E}-3$ & $1.921 \mathrm{E}-3$ & $5.656 \mathrm{E}-4$ & $1.498 \mathrm{E}-4$ & $3.766 \mathrm{E}-5$ & $9.366 \mathrm{E}-6$ & $2.286 \mathrm{E}-6$ \\
\hline Conv. rate & - & 1.65 & 1.76 & 1.92 & 1.99 & 2.01 & 2.03 \\
\hline
\end{tabular}

Table 1: Constant mobility $M(u) \equiv 1$ : numerical errors and temporal convergence rates with periodic BC.

\begin{tabular}{|}
\hline$\Delta t$ & $6.4 \times 10^{-3}$ & $3.2 \times 10^{-3}$ & $1.6 \times 10^{-3}$ & $8 \times 10^{-4}$ & $4 \times 10^{-4}$ & $2 \times 10^{-4}$ & $1 \times 10^{-4}$ \\
\hline \hline \multicolumn{7}{|c|}{ ss-Euler } \\
\hline Rela. ener. error & $5.296 \mathrm{E}-3$ & $3.113 \mathrm{E}-3$ & $1.771 \mathrm{E}-3$ & $9.326 \mathrm{E}-4$ & $4.949 \mathrm{E}-4$ & $2.597 \mathrm{E}-4$ & $1.345 \mathrm{E}-4$ \\
\hline Conv. rate & - & 0.77 & 0.81 & 0.92 & 0.91 & 0.93 & 0.95 \\
\hline Rela. $L^{2}$ error & $4.052 \mathrm{E}-2$ & $2.936 \mathrm{E}-2$ & $1.957 \mathrm{E}-2$ & $1.078 \mathrm{E}-2$ & $5.812 \mathrm{E}-3$ & $3.093 \mathrm{E}-3$ & $1.621 \mathrm{E}-3$ \\
\hline Conv. rate & - & 0.47 & 0.59 & 0.86 & 0.90 & 0.91 & 0.93 \\
\hline \multicolumn{7}{|c|}{ cETDMs1 } \\
\hline Rela. ener. error & $2.255 \mathrm{E}-3$ & $9.229 \mathrm{E}-4$ & $4.546 \mathrm{E}-4$ & $2.520 \mathrm{E}-4$ & $1.596 \mathrm{E}-4$ & $1.112 \mathrm{E}-4$ & $6.581 \mathrm{E}-5$ \\
\hline Conv. rate & - & 1.29 & 1.02 & 0.85 & 0.67 & 0.52 & 0.76 \\
\hline Rela. $L^{2}$ error & $2.737 \mathrm{E}-2$ & $1.686 \mathrm{E}-2$ & $7.842 \mathrm{E}-3$ & $3.871 \mathrm{E}-3$ & $2.248 \mathrm{E}-3$ & $1.396 \mathrm{E}-3$ & $7.998 \mathrm{E}-4$ \\
\hline Conv. rate & - & 0.70 & 1.10 & 1.02 & 0.784 & 0.69 & 0.80 \\
\hline \multicolumn{7}{|c|}{ cETDMs2 } \\
\hline Rela. ener. error & $3.462 \mathrm{E}-5$ & $1.142 \mathrm{E}-5$ & $4.009 \mathrm{E}-6$ & $1.304 \mathrm{E}-6$ & $4.131 \mathrm{E}-7$ & $1.473 \mathrm{E}-7$ & $5.036 \mathrm{E}-8$ \\
\hline Conv. rate & - & 1.60 & 1.51 & 1.62 & 1.65 & 1.49 & 1.55 \\
\hline Rela. $L^{2}$ error & $1.358 \mathrm{E}-3$ & $4.112 \mathrm{E}-4$ & $1.344 \mathrm{E}-4$ & $4.432 \mathrm{E}-5$ & $1.628 \mathrm{E}-5$ & $4.842 \mathrm{E}-6$ & $1.759 \mathrm{E}-6$ \\
\hline Conv. rate & - & 1.72 & 1.61 & 1.60 & 1.45 & 1.75 & 1.46 \\
\hline
\end{tabular}

Table 2: Symmetric degenerate mobility $M(u)=\left|1-u^{2}\right|$ : numerical errors and temporal convergence rates with periodic BC.

\subsubsection{Coarsening rates}

The energy (2) is a phenomenological description of the interfacial energy for a two-phase system. Coarsening phenomena characterized by a spatial-temporal power law are often observed in such a system. After some initial transient stages that may be related to spinodal decomposition 


\begin{tabular}{|c|c|c|c|c|c|c|c|}
\hline$\Delta t$ & $6.4 \times 10^{-3}$ & $3.2 \times 10^{-3}$ & $1.6 \times 10^{-3}$ & $8 \times 10^{-4}$ & $4 \times 10^{-4}$ & $2 \times 10^{-4}$ & $1 \times 10^{-4}$ \\
\hline \multicolumn{8}{|c|}{ ss-Euler } \\
\hline Rela. ener. error & $7.396 \mathrm{E}-3$ & $5.385 \mathrm{E}-3$ & $3.690 \mathrm{E}-3$ & $2.126 \mathrm{E}-3$ & $1.102 \mathrm{E}-3$ & $5.109 \mathrm{E}-4$ & $2.413 \mathrm{E}-4$ \\
\hline Conv. rate & - & 0.46 & 0.55 & 0.80 & 0.95 & 1.11 & 1.08 \\
\hline Rela. $L^{2}$ error & $6.658 \mathrm{E}-2$ & $5.492 \mathrm{E}-2$ & $4.076 \mathrm{E}-2$ & $2.551 \mathrm{E}-2$ & $1.361 \mathrm{E}-2$ & $6.438 \mathrm{E}-3$ & $3.205 \mathrm{E}-3$ \\
\hline Conv. rate & - & 0.28 & 0.43 & 0.68 & 0.91 & 1.08 & 1.01 \\
\hline \multicolumn{8}{|c|}{ cETDMs1 } \\
\hline Rela. ener. error & $6.170 \mathrm{E}-3$ & $3.866 \mathrm{E}-3$ & $1.872 \mathrm{E}-3$ & $8.704 \mathrm{E}-4$ & $4.028 \mathrm{E}-4$ & $2.076 \mathrm{E}-4$ & $1.101 \mathrm{E}-4$ \\
\hline Conv. rate & - & 0.67 & 1.05 & 1.10 & 1.11 & 0.96 & 0.92 \\
\hline Rela. $L^{2}$ error & $6.054 \mathrm{E}-2$ & $4.296 \mathrm{E}-2$ & $2.310 \mathrm{E}-2$ & $1.091 \mathrm{E}-2$ & $5.145 \mathrm{E}-3$ & $2.767 \mathrm{E}-3$ & $1.516 \mathrm{E}-3$ \\
\hline Conv. rate & - & 0.50 & 0.90 & 1.08 & 1.08 & 0.90 & 0.87 \\
\hline \multicolumn{8}{|c|}{ cETDMs2 } \\
\hline Rela. ener. error & $9.848 \mathrm{E}-4$ & $1.863 \mathrm{E}-4$ & $1.664 \mathrm{E}-5$ & $3.926 \mathrm{E}-6$ & $8.194 \mathrm{E}-7$ & $1.205 \mathrm{E}-7$ & $2.692 \mathrm{E}-8$ \\
\hline Conv. rate & - & 2.40 & 3.49 & 2.08 & 2.26 & 2.77 & 2.16 \\
\hline Rela. $L^{2}$ error & $2.855 \mathrm{E}-2$ & $5.972 \mathrm{E}-3$ & $7.051 \mathrm{E}-4$ & $1.915 \mathrm{E}-4$ & $5.044 \mathrm{E}-5$ & $1.319 \mathrm{E}-5$ & $3.475 \mathrm{E}-6$ \\
\hline Conv. rate & - & 2.26 & 3.08 & 1.88 & 1.92 & 1.93 & 1.92 \\
\hline
\end{tabular}

Table 3: One-sided degenerate mobility $M(u)=|1+u| / 2$ : numerical errors and temporal convergence rates with periodic BC.

or phase nucleation processes, the system become dominated by the coarsening process with a characteristic length scale $l$ that grows typically like a power of time

$$
l(t)^{\alpha}=l(0)^{\alpha}+c t
$$

for some positive constants $c$ and $\alpha$. Notice that the free energy of the Cahn-Hilliard equation is proportional to the area of the interface which has a dimension of $l^{-1}$. Consequently we expect

$$
\ln \mathcal{E}=-\frac{1}{\alpha} \ln t+C+o(1)
$$

when $t$ goes to infinity.

The energy evolutions of the solutions, $\ln$ (Energy) vs. $\ln$ (Time), for the periodic boundary condition problem are plotted in Figure 3 and those for the no-flux boundary condition problem are again similar. All curves are pretty close to straight lines except the case of the cETDMs2 scheme with the largest time step size $\Delta t=6.4 \times 10^{-3}$ for $M(u)=|1+u| / 2$, where the curve shows more visible oscillations while being monotonically decreasing. We do linear regression to determine the constant $A=-1 / \alpha$ and present the results in Table 4 for the different cases using different time step sizes. We can easily observe fast convergence of simulated coarsening exponents produced by the cETDMs2 scheme even with very large time step sizes. On the other hand, the first-order schemes, ss-Euler and cETDMs1 are only accurate when the time step size gets much smaller. We note that these values are computed over a relative short time interval only for convergence tests. The long time statistics are reported later through more extensive simulations.

\subsection{Coarsening dynamics under different diffusion mobilities}

By utilizing the efficiency and accuracy of the cETDMs2 scheme with large time step sizes, in this section we numerically simulate and investigate the coarsening dynamics in long-time phase separation processes under constant and degenerate mobilities and also their changes with respect to variations of the volume fraction $\phi$. We started the simulations at time $t=0$ with initial configurations being uniform state $\bar{u}=2 \phi-1$ plus random perturbation uniform in $[-0.2,0.2]$. 

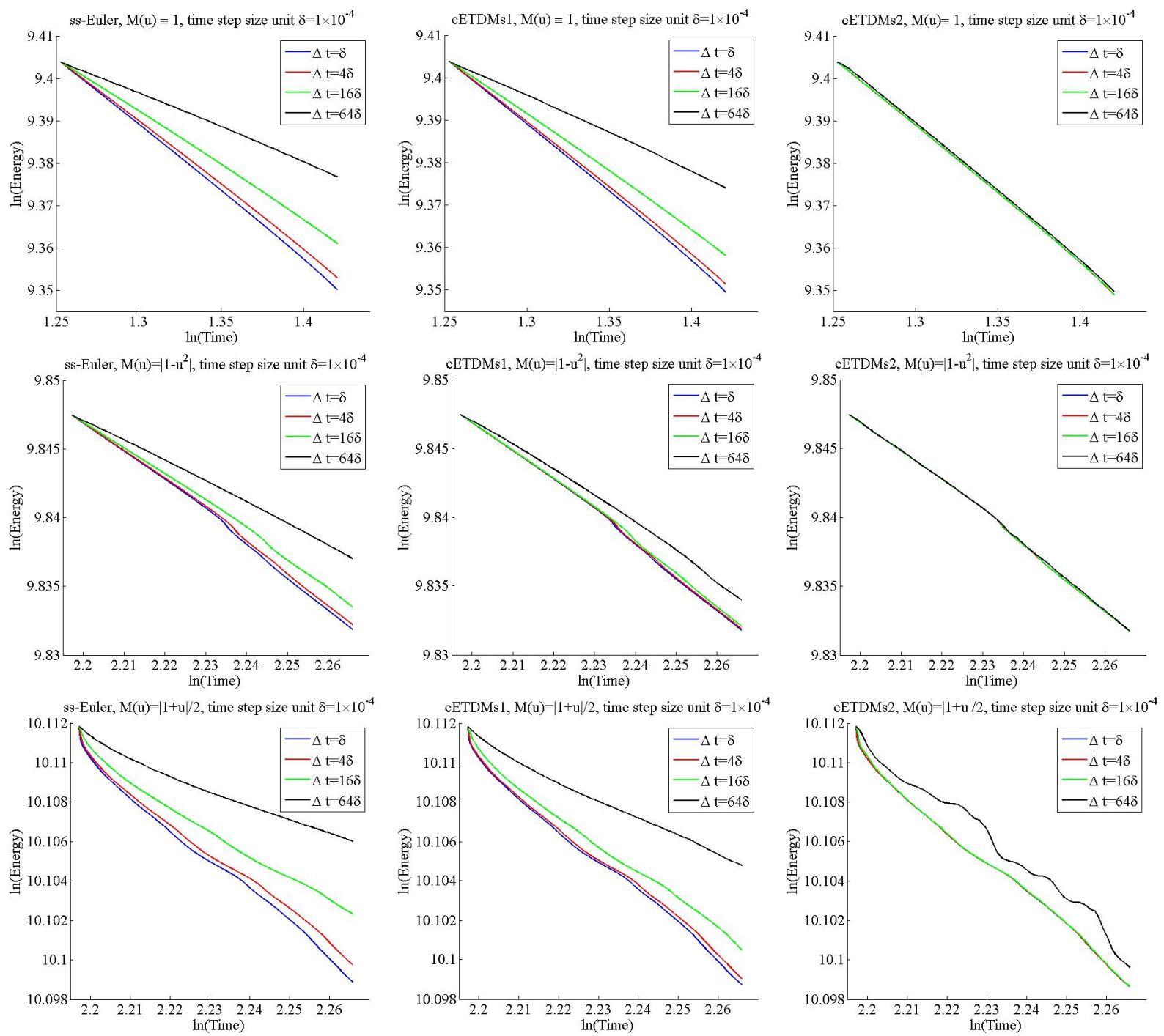

Figure 3: Plots of $\ln$ (Energy) vs. $\ln$ (Time) for all convergence test problems with periodic boundary condition. From top to bottom: $M(u) \equiv 1, M(u)=\left|1-u^{2}\right|$, and $M(u)=|1+u| / 2$. From left to right: ss-Euler, cETDMs1, cETDMS2.

For all three mobilities, we picked $\phi=0.25,0.5$ and 0.75 as representative cases of low, equal and high positive volume fractions for illustrations. The cases with other volume fractions are also interesting from the application point of view and they will be left for future studies.

\subsubsection{Constant mobility}

The coarsening rate for the constant mobility $M(u) \equiv 1$ is theoretically expected to be $-1 / 3$. The energy plots in Figure 3 and data given in Table 4 in short-period simulations in the preceding section also indicate such phenomena. Here we perform another 6 simulations for $\phi=0.25,0.5$ and 0.75 each, in which 3 are with periodic boundary condition and 3 with no-flux boundary condition. There are 18 simulations in total which all ended at $t=T=10$ with the time step size $\Delta t=5 \times 10^{-3}$. Some computed configurations at the final time with different volume fractions are shown in Figure 4. We also plot the energy evolution curves for two of the simulations with 


\begin{tabular}{|c||c|c|c|c|}
\hline$\Delta t$ & $6.4 \times 10^{-3}$ & $1.6 \times 10^{-3}$ & $4 \times 10^{-4}$ & $1 \times 10^{-4}$ \\
\hline \hline \multicolumn{5}{|c|}{ Constant mobility: $M(u) \equiv 1$} \\
\hline Ss-Euler & -0.1618 & -0.2556 & -0.3043 & -0.3211 \\
cETDMs1 & -0.1778 & -0.2724 & -0.3127 & -0.3241 \\
cETDMs2 & -0.3230 & -0.3275 & -0.3279 & -0.3279 \\
\hline \hline \multicolumn{5}{|c|}{ Symmetric degenerate mobility: $M(u)=\left|1-u^{2}\right|$} \\
\hline Ss-Euler & -0.1520 & -0.2033 & -0.2218 & -0.2271 \\
cETDMs1 & -0.1963 & -0.2139 & -0.2267 & -0.2281 \\
cETDMs2 & -0.2288 & -0.2290 & -0.2290 & -0.2290 \\
\hline \hline \multicolumn{5}{|c|}{ One-sided degenerate mobility: $M(u)=|1+u| / 2$} \\
\hline ss-Euler & -0.0844 & -0.1378 & -0.1755 & -0.1881 \\
cETDMs1 & -0.1023 & -0.1644 & -0.1858 & -0.1900 \\
cETDMs2 & -0.1780 & -0.1916 & -0.1916 & -0.1916 \\
\hline
\end{tabular}

Table 4: Convergence of the exponent $A=-1 / \alpha$ on the benchmark interval with periodic BC.

the volume fraction $\phi=0.75$ in Figure 5 , all the others are similar to them. To stay away from the initial transient dynamics, based on the energy plots, we chose the time interval $\left[e^{-2}, 10\right]$ to calculate the coarsening rates and the results are reported in Table 5. The results support the conclusion that the coarsening rates for all volume fractions are very close to $-1 / 3$ with small deviations.
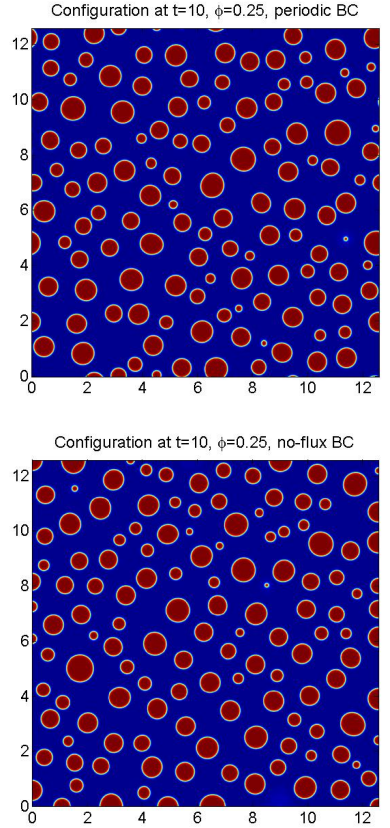
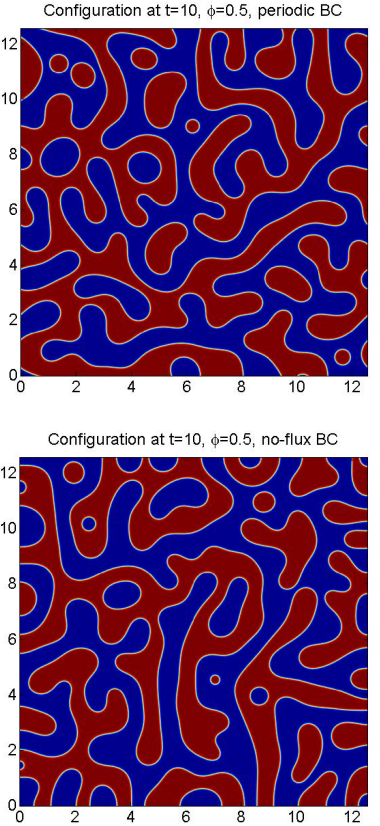

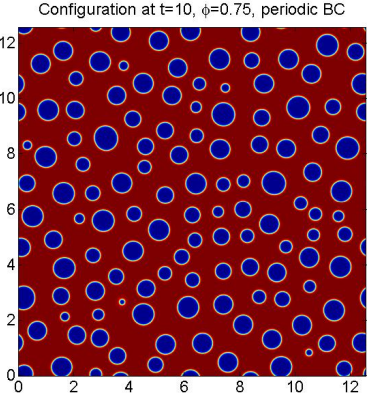

Configuration at $\mathrm{t}=10, \phi=0.75$, no-flux $\mathrm{BC}$

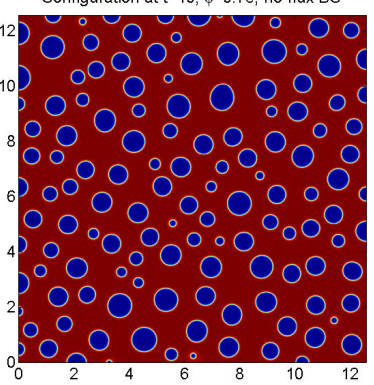

Figure 4: Constant mobility $M(u) \equiv 1$ : some final configurations at $T=10$ simulated by the cETDMs2 scheme. From left to right: $\phi=0.25,0.5,0.75$ respectively. Top: periodic BC; bottom: no-flux BC.

\subsubsection{Symmetric or two-sided degenerate mobility}

We next turn to the Cahn-Hilliard equation with the symmetric degenerate mobility $M(u)=$ $\left|1-u^{2}\right|$. Given the symmetry of the double well potential and the mobility function in this case, 


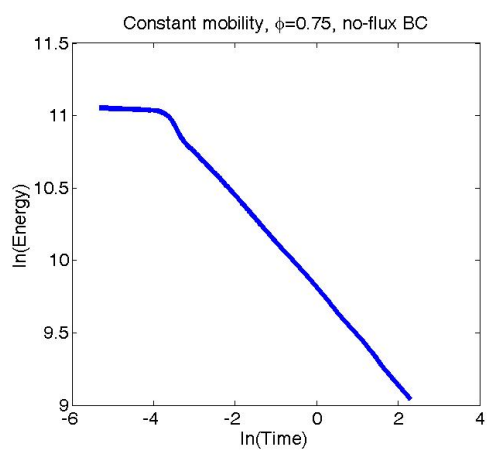

Figure 5: Constant mobility $M(u) \equiv 1$ : plots of $\ln$ (Energy) vs. $\ln ($ Time) in the time interval $[0,10]$ for two of the simulations with $\phi=0.75$ by the cETDMs2 scheme. Left: periodic BC; right: no-flux BC.

\begin{tabular}{|c||c|c|c|c|c|c|c|c|}
\hline \multicolumn{1}{|c||}{} & \multicolumn{4}{c|}{ Periodic BC } & \multicolumn{4}{c|}{ No-flux BC } \\
\hline \hline Coarsening rate & run1 & run2 & run3 & average & run1 & run2 & run3 & average \\
\hline$\phi=0.25$ & -0.2975 & -0.3437 & -0.3467 & $\mathbf{- 0 . 3 2 9 3}$ & -0.3094 & -0.3340 & -0.3626 & $\mathbf{- 0 . 3 3 5 3}$ \\
\hline$\phi=0.50$ & -0.2886 & -0.3015 & -0.3516 & $\mathbf{- 0 . 3 1 3 9}$ & -0.3384 & -0.3536 & -0.3192 & $\mathbf{- 0 . 3 3 7 1}$ \\
\hline$\phi=0.75$ & -0.3141 & -0.3431 & -0.3288 & $\mathbf{- 0 . 3 2 8 7}$ & -0.3450 & -0.3445 & -0.3109 & $\mathbf{- 0 . 3 3 3 5}$ \\
\hline
\end{tabular}

Table 5: Constant mobility $M(u) \equiv 1$ : coarsening rates simulated by the cETDMs2 scheme.

the coarsening rates for volume fraction $\phi$ and $1-\phi$ should be the same. We took the time step size $\Delta t=2 \times 10^{-3}$ and $T=40$. We ran totally 18 simulations using our method for $\phi=0.25,0.5,0.75$, again half with periodic boundary condition and the other half with no-flux boundary condition. The final configurations for three simulations with periodic boundary condition and different volume fractions are shown in Figure 6 and corresponding energy evolution curves in Figure 7. The data in the time interval $[1,40]$ are used to calculate all coarsening rates, again based on the energy plots to avoid the initial transient period, and the results are reported in Table 6. The simulation results clearly indicate the symmetry of the coarsening rate with respect to $\phi$. The coarsening rates for $\phi=0.25$ and $\phi=0.75$ are very close to each other with the averages being -0.1777 and -0.1763 respectively, if we do not discriminate periodic and no-flux boundary conditions. However, the coarsening for $\phi=0.5$ takes place faster and reaches an averaged rate of -0.2266 and this value close to theoretical predictions of a coarsening rate of -0.25 which is significantly lower than constant mobility cases.
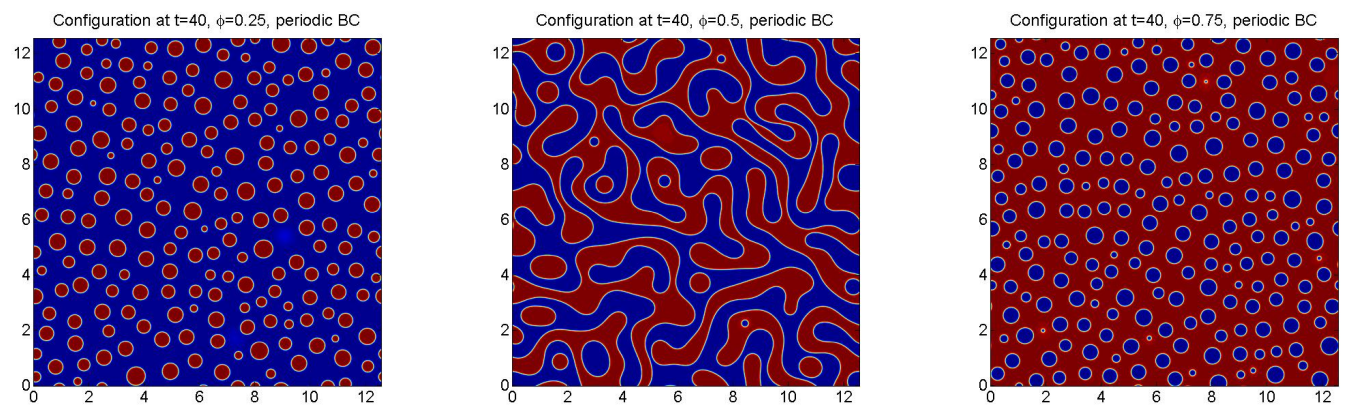

Figure 6: Symmetric degenerate mobility $M(u)=\left|1-u^{2}\right|$ : some final configurations with periodic BC at $t=40$ simulated by the cETDMs2 scheme. From left to right: $\phi=0.25,0.5,0.75$ respectively. 

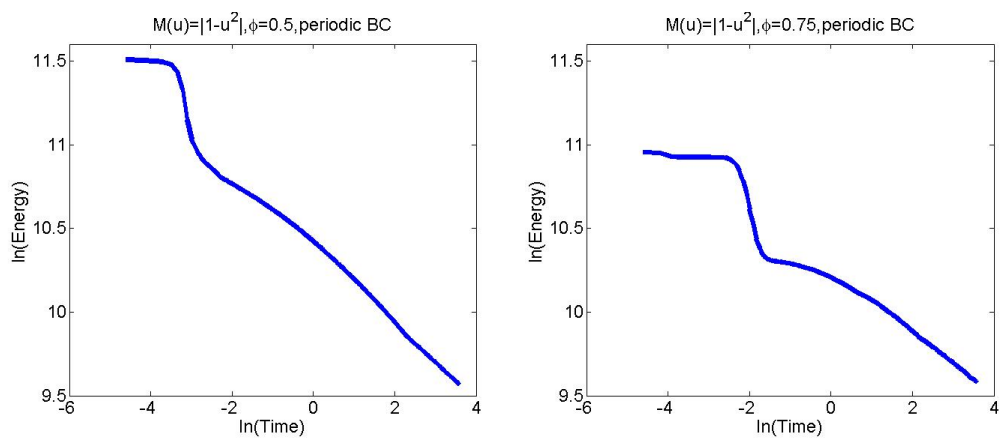

Figure 7: Symmetric degenerate mobility $M(u)=\left|1-u^{2}\right|$ : plots of $\ln$ (Energy) vs. $\ln ($ Time) in the time interval $[0,40]$ for some simulations with periodic BC by the cETDMs2 scheme. From left to right: $\phi=0.25,0.5,0.75$ respectively.

\begin{tabular}{|c||c|c|c|c|c|c|c|c|}
\hline \multicolumn{1}{|c||}{} & \multicolumn{4}{c|}{ Periodic BC } & \multicolumn{4}{c|}{ No-flux BC } \\
\hline \hline Coarsening rate & run1 & run2 & run3 & average & run1 & run2 & run3 & average \\
\hline$\phi=0.25$ & -0.1827 & -0.1736 & -0.1816 & $\mathbf{- 0 . 1 7 9 3}$ & -0.1796 & -0.1732 & -0.1759 & $\mathbf{- 0 . 1 7 6 2}$ \\
\hline$\phi=0.50$ & -0.2255 & -0.2215 & -0.2219 & $\mathbf{- 0 . 2 2 3 0}$ & -0.2232 & -0.2340 & -0.2335 & $\mathbf{- 0 . 2 3 0 2}$ \\
\hline$\phi=0.75$ & -0.1789 & -0.1749 & -0.1844 & $\mathbf{- 0 . 1 7 9 4}$ & -0.1736 & -0.1654 & -0.1805 & $\mathbf{- 0 . 1 7 3 2}$ \\
\hline
\end{tabular}

Table 6: Symmetric degenerate mobility $M(u)=\left|1-u^{2}\right|$ : coarsening rates simulated by the cETDMs2 scheme.

\subsubsection{One-sided degenerate mobility}

We now consider the one-sided degenerate mobility $M(u)=|1+u| / 2$. We expect different behaviors between the small positive volume fraction cases where the volume fraction $\phi$ is in the range of $[0,0.5)$ and the large positive volume fraction cases where $\phi$ is in $(0.5,1]$. We took the time step size $\Delta t=2 \times 10^{-3}$. We ran a total of 18 simulations for $\phi=0.25,0.5,0.75,3$ runs with periodic boundary condition and 3 with no-flux boundary condition for each $\phi$. The length of simulation time interval was set to be $T=80$ for $\phi=0.25$ and $T=10$ for $\phi=0.5$ and 0.75 . The final configurations for three simulations with periodic boundary condition and different volume fractions are shown in Figure 8 and corresponding energy evolution curves are in Figure 9. The energy plots clear indicate the transition from initial transient stages to later coarsening stages.

Since the simulations with different $\phi$ have different initial transient stages and enter the power law stage at very different time, the coarsening rates for $\phi=0.5$ and 0.75 are calculated on time interval $\left[e^{-1}, 10\right]$, and $[10,80]$ for $\phi=0.25$. The simulated results on coarsening rates are reported in Table 7. The results indicate that the coarsening rates for $\phi=0.5,0.75$ are close to $-1 / 3$, and get faster with decreasing $\phi$, which is similar to the constant mobility case. However, the coarsening processes for $\phi=0.25$ are much slower in comparison with other volume fractions and the averaged coarsening rate is about -0.2265 .

\begin{tabular}{|c||c|c|c|c|c|c|c|c|}
\hline \multicolumn{1}{|c||}{} & \multicolumn{4}{c|}{ Periodic BC } & \multicolumn{4}{c|}{ No-flux BC } \\
\hline \hline Coarsening rate & run1 & run2 & run3 & average & run1 & run2 & run3 & average \\
\hline$\phi=0.25$ & -0.2082 & -0.2455 & -0.2230 & $\mathbf{- 0 . 2 2 5 6}$ & -0.2306 & -0.2395 & -0.2118 & $\mathbf{- 0 . 2 2 7 3}$ \\
\hline$\phi=0.50$ & -0.3130 & -0.3080 & -0.3105 & $\mathbf{- 0 . 3 1 0 5}$ & -0.3193 & -0.3062 & -0.3170 & $\mathbf{- 0 . 3 1 4 2}$ \\
\hline$\phi=0.75$ & -0.2831 & -0.2928 & -0.2881 & $\mathbf{- 0 . 2 8 8 0}$ & -0.2916 & -0.2732 & -0.2950 & $\mathbf{- 0 . 2 8 6 6}$ \\
\hline
\end{tabular}

Table 7: One-sided degenerate mobility $M(u)=|1+u| / 2$ : coarsening rates simulated by the cETDMs2 scheme. 

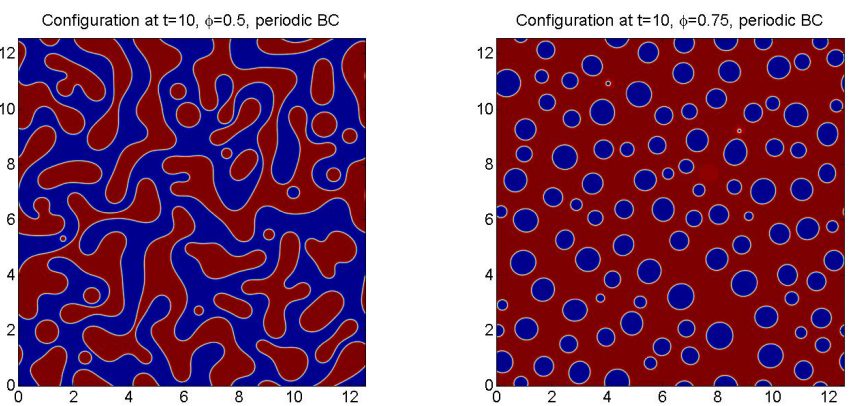

Figure 8: One-sided degenerate mobility $M(u)=|1+u| / 2$ : some final configurations with periodic BC simulated by the cETDMs2 scheme at $t=80$ for $\phi=0.25$ and at $T=10$ for $\phi=0.5,0.75$ respectively.
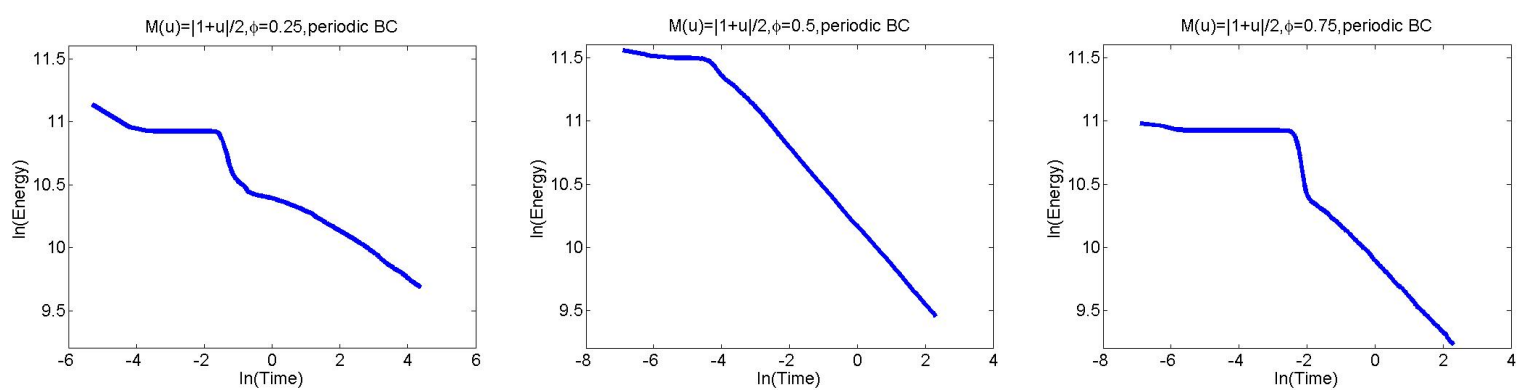

Figure 9: One-sided degenerate mobility $M(u)=|1+u| / 2$ : plots of $\ln$ (Energy) vs. $\ln$ (Time) for five of some simulations with periodic BC by the cETDMs2 scheme for $\phi=0.25$ in the time interval $[0,80]$ and $\phi=0.5,0.75$ respectively in the time interval $[0,10]$.

\section{Conclusions}

A fast and stabilized compact exponential time differencing multistep method is presented for solving the Cahn-Hilliard equation in a rectangular domain. It is demonstrated to have high order accuracy through numerical experiments. Moreover, even though it is explicit, the use of time splitting allows the use of large time steps for stable long-time integration. Meanwhile, the high order nature also alleviate the effect of splitting error associated with the low order time discretization. This assures stable and accuracy approximation of the long-time dynamics. The method can deal with various boundary conditions and can be implemented via FFT based computations. Though a compact central finite difference scheme is used for the spatial discretization, depending on the boundary conditions, Fourier spectral or Chebyshev spectral methods can also be used [29].

This newly developed algorithm allows us to study numerically the coarsening kinetics corresponding to different choices of the diffusion mobility with a far better spatial resolution and time accuracy than those in the literature before. We provided various estimates of the coarsening rates depending on the diffusion mobility and the volume fractions. The successful simulations in the two-dimensional domain give promises for studying similar problems in the three space dimensions which we plan to carry out in the near future. Applications to more complicated phase field models such as those incorporating anisotropic bulk elastic effects [34] and nucleation events [35] and high order interfacial curvature effects [36] will also be undertaken. 


\section{Acknowledgments}

The authors would like to thank Dr. Shibin Dai for fruitful discussions on the subject and the referees for their constructive comments that have helped us improving the manuscript.

\section{References}

[1] I.M. Lifshitz and V.V. Slyozov, The kinetics of precipitation from supersaturated solid solutions, J. Phys. Chem. Sol., 19 (1961), 35-50.

[2] C. Wagner, Theorie der Alterung von Niederschlagen dursch Umlosen, Z. Electrochem., 65 (1961), 581-594.

[3] J.W. Cahn and J.E. Hillard, Free energy of a nonuniform system I. Interfacial free energy, $J$. Chem. Phys., 28 (1958), 258-267.

[4] R.L. Pego, Front migration in the nonlinear Cahn-Hilliard equation, Proc. Royal Soc. London A, 442 (1989), 261-278.

[5] J.W. Cahn, C.M. Elliott, and A. Novick-Cohen, The Cahn-Hilliard equation with a concentration-dependent mobility: motion by minus the Laplacian of the mean curvature, European J. Appl. Math., 7 (1996), 287-301.

[6] S. Dai and Q. Du, Motion of interfaces governed by the Cahn-Hilliard equation with highly disparate diffusion mobility, SIAM J. Appl. Math., 72 (2012), 1818-1841.

[7] S. Dai and Q. Du, Coarsening mechanism for systems governed by the Cahn-Hilliard equation with degenerate diffusion mobility, Multi. Model. Simul., 12 (2014), 1870-1889.

[8] T.M. Rogers, K.R. Elder and R.C. Desai, Numerical study of the late stages of spinodal decomposition, Phys. Rev. B, 37 (1988), 9638-9649.

[9] T.M. Rogers and R.C. Desai, Numerical study of late-stage coarsening for off-critical quenches in the Cahn-Hilliard equation of phase separation, Phys. Rev. B, 39 (1989), 11956-11964.

[10] T. Kupper and N. Masbaum, Simulation of particle growth and Ostwald ripening via the Cahn-Hilliard equation, Acta.Metall. Mater., 42 (1994), 1847-1858.

[11] A.J. Bray and C.L. Emmott,, Lifshitz-Slyozov scaling for late-stage coarsening with an order parameter-dependent mobility, Phys. Rev. B, 52 (1995), R685-R688.

[12] J. Zhu, L.-C. Chen, J. Shen and V. Tikare, Coarsening kinetics from a variable-mobility CahnHilliard equation: Application of a semi-implicit Fourier spectral method, Phy. Rev. E, 60 (1999), 3564-3572.

[13] D. Fan, S. Chen, L.-Q. Chen and P. Voorhees, Phase-field simulation of 2-d Ostwald ripening in the high volume fraction regime, Acta Materialia, 50 (2002), 1895-1907.

[14] H. Garcke, B. Niethammer, M. Rumpf and U. Weikard, Transient coarsening behaviour in the Cahn-Hilliard model, Acta Materialia, 51 (2003), 2823-2830. 
15] R.V. Kohn and F. Otto, Upper bounds on coarsening rates, Comm. Math. Phys., 229 (2002), 375-395.

[16] L.-Q. Chen and J. Shen, Applications of semi-implicit Fourier-spectral method to phase field equations, Comput. Phys. Comm., 108 (1998), 147-158.

[17] S. Wise, J. Kim and J. Lowengrub, Solving the regularized, strongly anisotropic Cahn-Hilliard equation by an adaptive nonlinear multigrid method, J. Computat. Phys., 226 (2007), 414-446.

[18] W.M. Feng, P. Yu, S. Y. Hu, Z.-K. Liu, Q. Du and L.-Q. Chen, Spectral implementation of an adaptive moving mesh method for phase-field equations, J. Comput. Phys., 220 (2006), 498-510.

[19] W.M. Feng, P. Yu, S. Y. Hu, Z.-K. Liu, Q. Du and L.-Q. Chen, A Fourier spectral moving mesh method for the Cahn-Hilliard equation with elasticity, Comm. Comput. Phys., 5 (2009), 582-599.

[20] J. Shen and X. Yang, Numerical aproximations of Alen-Cahn and Cahn-Hiliard equations, Disc. Conti. Dyn. Sys.- A, 28 (2010), 1669-1691.

[21] J. Zhang and Q. Du, Numerical studies of discrete approximations to the Allen-Cahn equation in the sharp interface limit, SIAM J. Sci. Comput., 31 (2009), 3042-3063.

[22] L.Q. Chen, Phase-field models for microstructure evolution, Annual Review of Materials Science, 32 (2002), 113-140.

[23] D. Eyre, An unconditionally stable scheme for gradient systems, MRS Proceedings. Vol. 529, Cambridge University Press, 1998.

[24] C. Wang and S. Wise, An energy stable and convergent finite difference scheme for the modified phase field crystal equation, SIAM J. Numer. Anal., 49 (2011), 945-969.

[25] M. Hochbruck, C. Lubich and H. Selhofer, Exponential integrators for large systems of differential equations, SIAM J. Sci. Comput., 19 (1998), 1552-1574.

[26] S. Cox and P. Matthews, Exponential time differencing for stiff systems, J. Comput. Phys., 176 (2002, pp. 430-455.

[27] S. Krogstad, Generalized integrating factor methods for stiff PDEs, J. Comput. Phys., 203 (2005), 72-88.

[28] L. Ju, X. Liu and W. Leng, Compact implicit integration factor methods for a family of semilinear fourth-order parabolic equations, Dis. Cont. Dyn. Sys. B, 19 (2014), 1667-1687.

[29] Q. Du and W.-X. Zhu, Stability analysis and applications of the exponential time differencing schemes, J. Comput. Math., 22 (2004), 200-209.

[30] M. Hochbruck and A. Ostermann, Exponential integrators, Acta Numerica, 19 (2010), 209-286.

[31] C.M. Elliott and H. Garcke, On the Cahn-Hilliard equation with degenerate mobility, SIAM J. Math. Anal., 27 (1996), 404-423. 
[32] G. Sheng, T. Wang, Q. Du, K. Wang, Z. Liu and L.-Q. Chen, Coarsening kinetics of a two phase mixture with highly disparate diffusion mobility, Commun. Comput. Phys., 8 (2010), 249-264.

[33] L. Ju, J. Zhang, L. Zhu and Q. Du, Fast explicit integration factor methods for semilinear parabolic equations, J. Sci. Comput., 62 (2015), 431-455.

[34] S.-Y. Hu, Phase-field models of microstructure evolution in a system with elastic inhomogeneity and defects, PhD Diss, The Pennsylvania State University, 2004.

[35] T. Heo, L. Zhang, Q. Du and L.-Q. Chen, Incorporating diffuse-interface nuclei in phase-field simulations, Scripta Mater., 63 (2010), 8-11.

[36] Q. Du, C. Liu and X. Wang, A phase field approach in the numerical study of the elastic bending energy for vesicle membranes, J. Comput. Phys., 198 (2004), 450-468. 